\title{
Tentativa de crianças em lidar com as emoções de raiva e tristeza
}

\author{
Maria da Graça Bompastor Borges Dias \\ Universidade Federal de Pernambuco \\ Arne Vikan \\ Norges teknisk-naturvitenskapelige universitet - NTNU \\ Sissel Gravås \\ Norges teknisk-naturvitenskapelige universitet - NTNU
}

Resumo

O presente estudo focaliza respostas de crianças sobre como parar o sentimento de tristeza e raiva, o que implica explorar estratégias de alterações de humor, i.e., pensamentos sobre como regular as emoções. Foi perguntado a crianças brasileiras e norueguesas de 5, 7 e 9 anos de idade como a tristeza e a raiva podiam cessar em 4 diferentes contextos para cada emoção. Os resultados mostram que as estratégias de regulação das emoções (RE) variavam em função do contexto e da idade; que as crianças brasileiras usaram mais a interação social e as crianças norueguesas recorreram mais às estratégias cognitivas para raiva, mas não para tristeza. Não foi observada uma diferença clara na estratégia de RE para tristeza e raiva.

Palavras-chave:

Lidando com emoções, sentimento de raiva, sentimento de tristeza

\footnotetext{
Abstract

Children trying to deal with emotions of anger and sadness The present study focus on children's cognitions of how to stop feeling sad and angry, which implies an exploration of their meta mood strategies, i.e. thoughts about how to regulate emotions. It was asking 5, 7, and 9-year-old Brazilian and Norwegian children how sadness and anger could be stopped in 4 different contexts for each emotion. The results showed
} 
Key words: dealing with emotions, anger, sadness. that ER strategies varied with context and age, that Brazilian children used more social interaction and Norwegian children more cognitive strategies for anger, but not for sadness, and that even three year old children could state different strategies in different emotion contexts. There was not observed a clear difference in ER strategy for sadness and anger. verbalizadas, podem ser centrais para o desenvolvimento da autoregulação de emoções em virtude do seu potencial para o controle emocional (Kopp, 1982; 1989; Sroufe, 1996).

A regulação emocional (RE) tem sido discutida em vários estudos (Eisenberg \& Fabes, 1992; Fox, 1994; Garber \& Dodge, 1991; Schore, 1994; Sroufe, 1996). É definida como “... os processos envolvidos na modificação de reações emocionais” (Oatley \& Jenkins, 1996, p. 192), e "comportamento visando mudanças de sentimentos numa direção desejada" (Saarni \& Crowley, 1990). Argumenta-se a necessidade em pesquisar como reduzir (ou aumentar) a intensidade da emoção expressa, retardar (ou aumentar a velocidade) seu início ou recuperação, o limite (ou aumento) de sua persistência em função do tempo, reduzir (ou aumentar) a extensão da emoção, e afetar outras características qualitativas de respostas de emoção (Thompson, 1994). Estas questões implicam que é o estado emocional mais do que sua exposição que deveria ser regulado (Terwogt \& Olthof, 1989; Thompson, 1990). 
Um amplo corpo de estudos nos diferentes aspectos da cognição da emoção de crianças (e.g., Harris, 1989), como também pesquisas em áreas relacionadas, tais como estratégias das crianças para lidar com atraso de gratificação (e.g., Mischel \& Mischel, 1983; Yates, Yates \& Beasley, 1987), dor (e.g., Reissland, 1983; Ross \& Ross, 1984; Curry \& Russ, 1985; Jerrett,1985; Branson \& Craig, 1988), estresse (Brown, O’Keeffe, Sanders \& Baker, 1986; Band \& Weisz,1988; Altshuler \& Ruble,1989), e seu foco para lidar com as emoções em geral, indica que crianças novas podem conceber estratégias que possibilitam-nas controlar eventos mentais emocionais, tais como o uso de vários tipos de distração.

Os resultados de vários estudos, que parecem ter focalizado as estratégias RE, são entretanto obscuros. Isto quando se considera o conhecimento de crianças das estratégias RE, as mudanças de idades que podem indicar desenvolvimento, e o significado de fontes de variação tais como tipo e contexto da emoção. Estes fatores podem fornecer informações sobre como o conhecimento da RE é organizado. Por exemplo, estudos com crianças de seis anos (Harris, Olthof \& Terwogt, 1981; Terwogt, Schene \& Harris, 1986) propõem que mudar a situação é melhor do que atuar na emoção. Isto ocorreu quando foi perguntado o que as crianças podiam fazer para ter ou não medo, raiva ou tristeza. Já crianças de quatro e cinco anos nos estudos de Kenealy (1989) e McCoy e Masters (1985) responderam com uma variedade de estratégias quando era perguntado o que elas faziam quando estavam infelizes, ou o que elas podiam fazer para fazer outra criança não sentir felicidade, tristeza e raiva.

Com o aumento da idade verifica-se o aumento de mudança do estado mental (Harris et al., 1981), de estratégias verbais e sociais (McCoy \& Masters, 1985) e de como evitar certas emoções através do uso de divertimento ou comportamento reativo (Kenealy, 1989). Uma avaliação dos resultados fica difícil não só pela utilização de diferentes categorias de respostas que foram empregadas, mas também em virtude dos testes ambíguos utilizados para detectar as estratégias RE. Questões sobre como evitar uma emoção podem se referir apenas a uma estratégia específica (evitação), enquanto descrições sobre o 
que as crianças fazem quando estão infelizes e quando têm o objetivo de influenciar outra criança, podem necessitar de habilidades de autoobservação, memória para o próprio comportamento e conhecimento de melhores estratégias de influência social e não das estratégias RE.

No entanto, o foco nas qualidades diferenciais das emoções na teoria evolucionista e na teoria cognitiva da emoção (e.g., Lazarus, 1991) resultou numa tentativa em testar a expectativa de que as estratégias de regulação estão relacionadas aos tipos de emoção. Esta expectativa tem sido apoiada de certo modo em estudos com adultos (Tice \& Baumeister, 1993), e em estudo com crianças mais velhas (Vikan \& Dias, 1996). O último estudo mostrou que técnicas cognitivas tais como: "Não pense sobre o fato" e "Pense que ele não é real", foram propostas para cessar o medo numa maior amplitude do que para cessar raiva e tristeza. No entanto, as perguntas utilizadas não estavam relacionadas a um contexto de respostas emocionais.

O tipo de contexto tem sido observado como influenciando estratégias emocionais em diversos estudos (Altshuler \& Ruble, 1989; Band \& Weisz, 1988; McCoy \& Masters, 1985), e o controle do contexto parece determinar a escolha em lidar com problema (baixo-controle) ou lidar com a emoção focalizada (autocontrole) (Altshuler \& Ruble, 1989). Assim, variações dos tipos de emoção podem ser consideradas erroneamente como variações relacionadas ao contexto quando este não é especificado.

O presente estudo teve como objetivo esclarecer o uso das estratégias RE entre crianças, mudanças em relação à idade que possam indicar desenvolvimento de estratégias, e as contribuições relativas ao tipo de emoção e contexto na variação das estratégias RE. O teste da estratégia RE foi apresentado a partir de uma questão de como uma protagonista (uma criança) de um desenho, em um dos contextos das quatro emoções para tristeza ou raiva, podia cessar a tristeza ou a raiva. Em estudo anterior (Vikan \& Clausen, 1994) foram usados os contextos que representavam as quatro causas mais freqüentemente mencionadas dessas duas emoções.

Devido à escassez de pesquisas anteriores, apenas expectativas gerais sobre a ocorrência de variações nas estratégias da emoção poderiam ser relacionadas ao tipo de emoção e contexto (e.g., Altshuler 
\& Ruble, 1989; Banda \& Weisz, 1988; McCoy \& Masters, 1985). Além disso, outros estudos sugerem um aumento do uso de estratégias cognitivas com idade (e.g., Altshuber \& Ruble, 1989; Band \& Weisz, 1988; Harris et al., 1981; Yates et al., 1987). E o estudo de Vikan e Dias (1996), demonstrou que crianças brasileiras, quando comparadas às norueguesas, apresentam uma freqüência maior de estratégias de interação social e de brincar e uma freqüência mais baixa de estratégias cognitivas.

\section{Método}

\section{Sujeitos}

Uma amostra de 288 crianças norueguesas e brasileiras de ambos os sexos, de grupos de idade de quatro, cinco anos (idade média, 5 anos e 3 meses), sete anos (idade média, 7 anos e 4 meses) e nove anos (idade média, 9 anos e 5 meses) de escolas de áreas urbanas que atendem a classe média da cidade do Recife e de escolas da Noruega.

\section{Contextos de emoção e materiais}

Desenhos mostrando uma criança no contexto da emoção. Os desenhos envolvendo os contextos para tristeza, como definido juntamente com as instruções, foram: 1 . Perdendo uma bola num rio; 2 . Leito de morte do avô; 3. Ser desprezado pelos amigos; 4. Repreendido pela mãe. Os contextos para raiva foram: 1 . Ser zombado por amigos; 2. Discutir com um colega; 3. Seu livro ser rasgado por uma criança menor; 4. O pai proibir o colega de brincar junto. Cada desenho mostrava a criança com a emoção (tristeza ou raiva) correspondendo ao contexto. O sexo do protagonista nos desenhos combinava com o sexo da criança entrevistada.

\section{Procedimento e questões experimentais}

Os sujeitos foram testados individualmente numa sala na própria escola. Antes do teste, o experimentador (E) informava à criança que lhe seriam feitas algumas perguntas para saber como ela pensa sobre estar triste e zangada. Era dito que crianças podiam ter todas as espécies de emoções, como alegria, tristeza, raiva, medo etc., e que o $\mathrm{E}$ 
sabia que o sujeito tinha estado triste ou zangado muitas vezes. O E, além do mais, dizia que os adultos várias vezes esqueciam como as crianças experienciavam emoções, e que ele desejava perguntar aos sujeitos alguma questão sobre estas emoções. Eram feitas, então, as duas primeiras questões. À metade dos sujeitos, foi perguntado primeiramente sobre raiva e à outra metade, sobre tristeza.

1. Como você se sente quando está triste/zangado?

2. Você sente o mesmo toda vez?

O experimentador dizia que iria mostrar um desenho de uma menina/menino que estava triste/zangado e mostrava o desenho do contexto que estava descrito nas três sentenças (i.e., "Aqui você vê uma menina que está triste. E aqui você vê porque ela está triste. Ela perdeu sua bola no rio"). Seguiam-se então mais três questões.

3. Quanto tempo você pensa que ela ficará triste?

Era pedido ao sujeito para indicar se ele achava que a emoção duraria por muitos dias, um dia inteiro, algumas horas, uma hora ou apenas um momento.

4. O que você acha que ela pode fazer para parar de ficar triste/ zangada?

5. Alguém pode fazer alguma coisa para fazê-la parar de ficar triste/zangada?

O E dizia que ele também desejava fazer algumas perguntas sobre a própria criança quando estava zangada/triste.

O procedimento para as questões de 3 a 5 foi então repetido para as questões 6 a 8 que foram direcionadas à criança e não mais a protagonista. Assim, foram feitas aos sujeitos um total de oito perguntas.

A seqüência das emoções e as combinações dos contextos foram balanceadas para cada amostra dos dois países. As respostas para todas as perguntas foram gravadas e transcritas literalmente, exceto respostas às questões 3 e 6 , as quais foram gravadas como um número na escala padrão de zero a cinco. A gravação da resposta foi iniciada quando a criança começava a responder.

Computação dos dados

As respostas à questão 1 sobre a experiência da emoção foram computadas quando nomeavam uma emoção (1), descrições de emo- 
ções ou estados emocionais (2), e outras (3). As respostas às questões 3 e 6 sobre duração foram contadas como um número correspondente à escala de zero a cinco de alternativas, com a alternativa mais longa (muitos dias) no número máximo de 5 . Já as respostas às perguntas 5 e 8 sobre apoio social, foram computadas como pais (1), amigos e outros (3).

As respostas às perguntas 4 e 7 sobre cessar/parar emoções foram originalmente contadas em treze categorias diferentes, que podem ser reduzidas às cinco seguintes:

1. Não-funcional. Recusas, respostas não contadas e "não sei".

2. Mudança ambiental. Respostas sugerindo atos que produzem mudanças no ambiente e não na emoção (e.g., "Dar ao avô uma droga milagrosa", "Comprar uma bola nova").

3. Interação social. Respostas do tipo "falando com outros", "confrontando outros", "desculpando-se aos outros", "estar com outros" ou "jogar com outros".

4. Atividades de distração. Construir ou produzir alguma coisa, brincadeira solitária ou cessar um comportamento (e.g., "Parar de brigar").

5. Técnicas cognitivas. Tentativas de modificar diretamente uma emoção (e.g., "Torna-se feliz", "Não levar os fatos a sério"), focalizando um estímulo (e.g., "Pensar em alguma coisa que a faça feliz"), reassegurando (e.g., "Pensar que o fato não significa nada"), desvio (e.g., "Pensar sobre outra coisa") e supressão (e.g., "Esquecer o fato", "Não pensar sobre ele").

Os números das categorias podem também ser concebidos como correspondentes a uma seqüência de desenvolvimento e, conseqüentemente, uma escala ordinal. A colocação das duas primeiras e da última categoria pode ser preferivelmente direta desde que as duas primeiras sugerem simples formas de evitar o problema, enquanto a última categoria se refere ao uso da cognição. As categorias 3 e 4 sugerem respostas mais maduras do que as duas primeiras categorias e geralmente evocam respostas menos desenvolvidas do que as técnicas cognitivas da categoria 5.

Desde que a concordância intra-avaliador no escore das categorias originais foi em torno de $90 \%$ para o escore de $25 \%$ das respostas no estudo anterior (Vikan \& Clausen, 1994), e para 25\% das respostas 
norueguesas no presente estudo $(n=144)$, nenhum outro exame de confiança foi julgado necessário para as cinco categorias aqui relatadas.

\section{Resultados}

As distribuições de respostas de escala ordinal para as questões sobre regulação da emoção e duração da emoção (questões 3, 4, 6 e 7) foram examinadas com uma análise de variância de Kruskal-Wallis e as diferenças de respostas para as outras questões foram avaliadas com o teste de U de Mann-Whitney, qui-quadrado de Pearson e teste Wilcoxon. As distribuições de respostas de escala ordinal para as questões sobre da regulação da emoção e duração da emoção (questões 3, 4, 6 e 7) foram examinadas com uma análise de variância de Kruskal-Wallis e as diferenças de respostas para as outras questões foram avaliadas com o teste de U de Mann-Whitney, qui-quadrado de Pearson e teste Wilcoxon.

Uma vez que os contextos naturalmente eram diferentes para as diferentes emoções, o esquema do estudo pode ser descrito como dois experimentos paralelos que se adaptavam a um teste fatorial de variação independente 2 (país) x 2 (sexo) x 3 (grupo de idade) x 4 (contexto) com medidas repetidas nos dois últimos fatores, e regulação da emoção de tristeza ou raiva como variáveis dependentes.

Nenhuma das distribuições de respostas apresentou diferenças relativas ao sexo.

\section{Regulação de emoção}

A Tabela 1 mostra a distribuição de respostas para as questões de cessar a tristeza e a raiva.

A tabela mostra variações relacionadas aos grupos de país e idade, mas não relacionadas ao contexto, que parecia ser a mais proeminente fonte de variação. As análises de Kruskal-Walls mostraram variações relacionadas ao grupo de idade, $X^{2}(4)=26.56$, e ao contexto, $\mathrm{X}^{2}(4)=39.86$, ambos $\mathrm{p}<0.001$ para tristeza, e variação relacionada a grupo de idade, $X^{2}(4)=11.0, p=0.025$, país, $X^{2}(4)=34.99$, $p<0.001$ e contexto $X^{2}(4)=14,81, p<0.01$ para raiva. Pode-se sugerir que a diferença de idade sustenta a classificação das categorias 3 e 4 . O teste não 
Tabela 1

Percentagens de respostas por categorias de regulação da emoção para tristeza e raiva

por país e grupo de idade ( $n=144$ para cada amostra de emoção e $n=48$ para cada grupo

de idade).

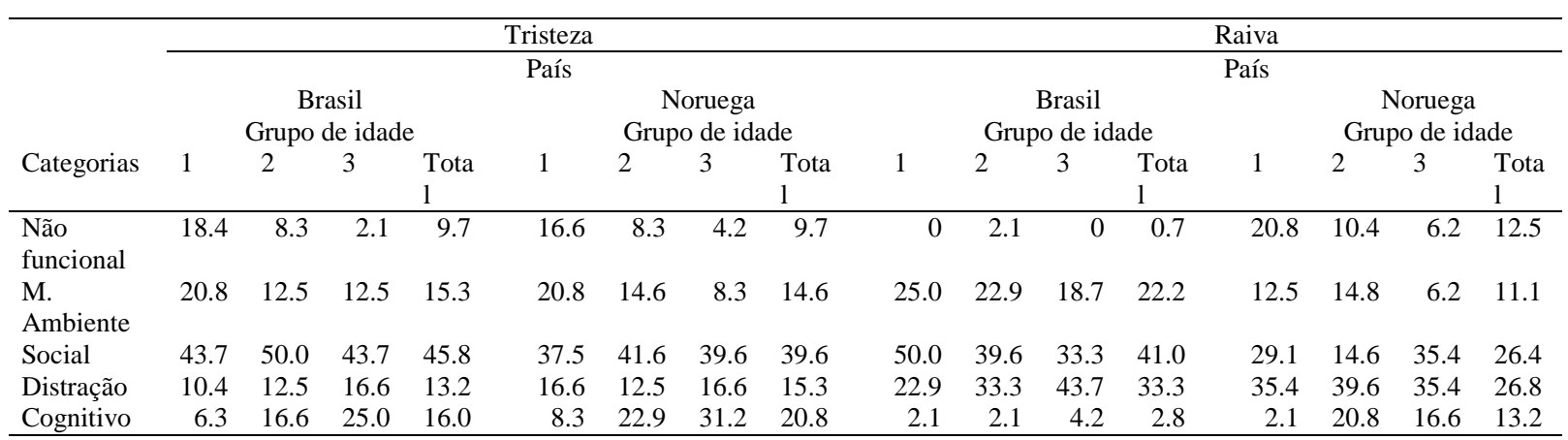


mostrou uma diferença significativa concernente à idade quando a classificação relativa dessas duas categorias estava invertida. Testes separados de Wilcoxon para as amostras brasileira e norueguesa não mostraram diferenças significativas para tipo de emoção.

Como é sugerido na Tabela 1 , as variações de grupo de idade geralmente estão relacionadas a uma diminuição na frequiência de categorias não-funcional e um aumento de freqüência das cognitivas para tristeza e raiva. Testes para diferenças de nível (U de MannWhitney) sugeriram que a maior mudança foi entre os dois grupos de idade mais novos. Assim, os testes mostraram um $\mathrm{Z}=-2.85$, $\mathrm{p}<0.001$ para a diferença entre o grupo de 5 a 7 anos e $Z=-2.03$, p=0.04 para a diferença entre o grupo de 7 e 9 anos.

A Tabela 1 sugere ainda que a variação relativa ao país para raiva foi devida às frequiências mais altas entre as norueguesas e respostas não funcionais em todos os grupos de idade. Também devido às freqüências mais altas de respostas cognitivas das norueguesas nos dois grupos de idade mais velhos, enquanto as brasileiras mostraram freqüências mais altas de respostas sociais nos dois grupos de idade mais novos.

O relacionamento entre contextos e estratégias de regulação está indicado na Tabela 2, que mostra as freqüências de respostas em categorias por contextos.

Os quatro contextos de tristeza que são mostrados primeiro na tabela, evocam respostas de distribuições diferentes. Os testes de U de Mann-Whitney mostraram as menores diferenças entre perder a bola no rio e ser repreendido pela mãe, $Z=-2.02$, $p=0.043$, e ser repreendido pela mãe e ser posto de lado pelos amigos, $Z=-2,20$, $p=0.027$. Todas as outras diferenças corresponderam a $\mathrm{p}=0.008$ ou menor. Os quatro contextos de raiva podem parecer mais homogêneos, o que é apoiado pelos testes. Ser zombado por amigos evocou respostas diferentes do que ter o livro rasgado, $\mathrm{Z}=-2.87, \mathrm{p}<0.01$, e estar discutindo, $\mathrm{Z}=-2.42$, $\mathrm{p}=0.016$. Já o contexto de ter seu livro rasgado obteve respostas diferentes de ser proibido pelo pai, $\mathrm{Z}=-2.23$, $\mathrm{p}=0.026$.

A Tabela 2 mostra diversos exemplos de freqüências de respostas extremas. As respostas ambientais foram muito freqüientes, por exemplo, nos contextos de perda da bola, de discussão e do livro rasgado. 


\section{Tabela 2}

Percentagens de respostas por categorias de regulação da emoção em função dos contextos. $\mathrm{T}=$ tristeza, $\mathrm{R}=$ raiva, $\mathrm{n}=72$ para cada contexto.

Categorias de regulação da emoção

\begin{tabular}{lcccccc}
\hline \multicolumn{1}{c}{ Contexto } & $\begin{array}{c}\tilde{N}- \\
\text { Func }\end{array}$ & $\begin{array}{c}\text { M. } \\
\text { Ambie }\end{array}$ & $\begin{array}{c}\text { Soci } \\
\text { al }\end{array}$ & $\begin{array}{c}\text { Distraç } \\
\text { ão }\end{array}$ & Cognição & Total \\
\hline T: Perder a bola & 2.7 & 52.8 & 22.2 & 6.9 & 15.3 & 99.9 \\
T: Avô & 16.6 & & 25.0 & 19.4 & 38.9 & 99.9 \\
T: Escantear & 5.6 & 1.4 & 62.5 & 19.4 & 11.1 & 100.0 \\
T: Ralhar & 13.9 & 5.6 & 61.1 & 11.1 & 8.3 & 100.0 \\
R: Zombar & 11.1 & 2.8 & 29.2 & 41.6 & & 100.0 \\
R: Discutir & 1.4 & 33.3 & 22.2 & 40.3 & 2.8 & 100.0 \\
R: Rasgar & 13.9 & 23.6 & 27.8 & 25.0 & 9.7 & 100.0 \\
livro & & & & & & \\
R: Proibição do & & 6.9 & 55.6 & 33.3 & 4.1 & 99.9 \\
pai & & & & & & \\
\hline
\end{tabular}

As respostas foram, geralmente, para o efeito de que a tristeza poderia ser finalizada pelo ganho de uma nova bola, ou que a raiva poderia ser cessada pela conquista de um novo amigo ou ganho de outro livro. As frequiências mais altas para o contexto de interação social de ser posto de lado, da repreensão e da proibição do pai pareceram relatar, em parte, para o retorno ou procura de novos amigos no contexto de ser posto de lado e a combinação dos apelos e desculpas em relação à mãe e ao pai nos outros dois contextos. Isto significa que, quando a mãe repreende a criança, esta pode desculpar-se a si mesma e parar de ficar triste e, quando o pai proíbe, ela pode apelar para uma reconsideração e, conseqüentemente, fazer com que cesse a raiva. As maiores freqüências de distração de respostas nos contextos de ser zombado e discutir foram geralmente devido às respostas que se referiam ao brincar, enquanto respostas cognitivas no contexto do avô tiveram, na maior parte, alguma variação na tentativa de esquecer. 
A interação social foi freqüentemente mais usada classificandose na categoria para tristeza $(n=104)$ e raiva $(n=91)$. Uma classificação mais distante destas respostas nas subcategorias mostrou que "brincando com outros" inclui respectivamente $25,9 \%$ e $22 \%$ de respostas de interação social para tristeza e raiva; "pedindo alguma coisa, pegando alguma coisa ou recebendo alguma coisa", incluíram respectivamente $27,9 \%$ e $26,4 \%$; "revidando" (somente respostas para os contextos de ser zombado e discutir) incluíram respectivamente $11,5 \%$ e $22 \%$ das respostas; "conversando com outro" (mais freqüentemente mãe e pai) incluíram respectivamente $19,2 \%$ e $11 \%$ das respostas; e "conquistando outro amigo" (somente respostas nos contextos de ser posto de lado e discutir) incluíram respectivamente $11,5 \%$ e $6,6 \%$ das respostas de interação social.

\section{Duração da emoção}

Uma checagem na relação de respostas de regulação da emoção para a intensidade da emoção foi realizada comparando-se a distribuição de respostas do escore mais baixo (um momento ou uma hora), ao escore mais alto (muitos dias ou o dia inteiro) na duração da emoção. Nenhuma diferença consistente pôde ser identificada.

A única diferença que aparece nas análises estatísticas foi a variação relacionada ao contexto de tristeza, $X^{2}(4)=52,61 \%, p<0,001$. Isto foi especificamente relacionado ao contexto da morte do avô, que teve maior duração (média 4,1) do que a tristeza referindo-se a um dos outros três contextos (média 2,5). A média para avaliação da duração da raiva foi 2.7 o que mostra que as crianças geralmente calculam para estas duas emoções um curto espaço de tempo. As percentagens das crianças que calcularam a duração de tristeza e raiva por somente um momento foram respectivamente 35,7 e 37,4.

\section{Apoio social para cessar emoções}

Não houve diferença relacionada à idade para respostas de apoio social e, portanto, a Tabela 3 mostra as freqüências das respostas das categorias de apoio social somente para país e tipo de emoção. As freqüências na tabela sugerem que os brasileiros quando comparado aos noruegueses, referem-se mais freqüentemente aos pais e menos 
aos amigos como apoio social para as duas emoções. Isto foi apoiado pelos testes de qui-quadrado para tristeza e raiva com $X^{2}(2)=13,72$, $\mathrm{p}<0,01$ para a primeira, e $X^{2}(2)=11,75, \mathrm{p}<0,01$ para a última. A tabela também sugere uma diferença nas distribuições das emoções relacionadas a uma freqüência mais alta de apoio social dos pais para tristeza do que para raiva, $X^{2}=35,83, \mathrm{p}<0,001$.

Os testes de qui-quadrado também mostraram variações relacionadas ao contexto de tristeza $X^{2}(6)=25,98, p=0,01$ e de raiva $X^{2}(6)=20,94$, $\mathrm{p}<0,01$. Para tristeza, isto foi especificamente devido ao contexto de ser posto de lado, no qual a maioria das respostas $(54,2 \%, \mathrm{n}=72)$ sugeriram os amigos como apoio social quando comparado à maioria de escolha dos pais nos outros três contextos. O contexto causal para

\section{Tabela 3}

Percentagens de respostas nas categorias para apoio social, em função da emoção e do país.

\begin{tabular}{|c|c|c|c|c|}
\hline \multicolumn{3}{|c|}{ Tristeza } & \multicolumn{2}{|c|}{ Raiva } \\
\hline \multicolumn{3}{|c|}{ País } & \multicolumn{2}{|c|}{ País } \\
\hline Categorias & Brasil & Noruega & Brasil & Noruega \\
\hline Pais & 58.3 & 41.7 & 51.4 & 31.9 \\
\hline Amigos & 21.5 & 41.7 & 28.5 & 43.7 \\
\hline Outro & 20.5 & 16.6 & 20.1 & 24.3 \\
\hline Total & 99.9 & 100.0 & 100.0 & 99.9 \\
\hline
\end{tabular}

raiva foi a proibição do pai, com a grande maioria das respostas mostrando uma escolha dos pais sobre os colegas como apoio social $(58,3 \%$ vs. $33,3 \%)$.

Uma percentagem de 90,6 $(\mathrm{n}=117)$ das "outras" categorias de respostas foram de respostas não contáveis (e.g., professores, outros adultos, a polícia) e respostas de que ninguém poderia ajudar. As percentagens de sujeitos que disseram que ninguém poderia ajudar 
ou que não sabiam foram $15,8 \%$ para tristeza e $13,9 \%$ para raiva $(n=144$ em ambos os exemplos).

\section{Experiência da emoção}

Acima de dois terços das respostas de experiência emocional para tristeza e raiva foram contados como "descrições". As únicas respostas que não foram descrições, decresceram com a idade, $X^{2}(4)=21,42$, $\mathrm{p}<0,001$. Uma maior diferenciação das descrições mostrou que 22,2\% das respostas de tristeza e 15,3\% das de raiva foram elaborações da mesma emoção; $16,7 \%$ para tristeza e $4,9 \%$ para raiva, mencionaram uma parte do corpo; $11,8 \%$ para tristeza e $6,9 \%$ para raiva foram referências de estar só (quase todas da amostra brasileira); e 38,9\% e $56,3 \%$ respectivamente poderiam somente ser contados como "outras referências psicológicas", que tiveram um número de respostas de 115 para tristeza e 110 para raiva.

\section{Discussão}

Sustentando as expectativas gerais sobre a estratégia de respostas RE, duas das observações mais salientes deste estudo foram que a grande maioria das crianças de 4 e 5 anos poderia propor as estratégias RE para tristeza e raiva, e que o contexto foi a maior fonte de variação para as respostas sobre duração da emoção e de apoio social.

Uma alta percentagem das estratégias propostas entre as crianças mais novas pode sugerir que crianças de idade entre 4 e 5 anos não representam o limite mais baixo para formulações da estratégia RE. De fato, observações que crianças de 3 anos podem mostrar o uso sofisticado de termos para emoção, diferenciam conhecimento dos estados emocionais, compreendem uma variedade de razões pessoais para reações emocionais (Harris et al., 1981), e sabem como confortar, chatear e zombar que podem ser usados para influenciar emoções nos outros (Dunn \& Brown, 1991). Estes dados sugerem que o conhecimento das estratégias de regulações como "brincar" e "ir para a mãe" podem estar presente nesta idade, i.e., mais de 2 anos a menos do que a média de idade das amostras mais novas no presente estudo. Esta 
hipótese foi testada no estudo de Sissel, Vikan e Dias (A. Vikan, comunicação pessoal, novembro de 1999) com crianças norueguesas de 3 a 4 anos. Neste estudo os resultados mostraram que estratégias podem ser formuladas para tristeza e raiva e variam com o contexto. As duas estratégias mais freqüentes foram distração e interação social. As respostas de distração referiam-se quase exclusivamente a "brincar", enquanto as respostas sociais dividiam-se igualmente entre "indo para um amigo" e "para a mãe". Isto foi similar às respostas no mesmo contexto das crianças do grupo de idade mais jovem do presente estudo. Assim, a variação do contexto mostrou que até mesmo crianças mais novas podem selecionar estratégias RE de acordo com o contexto.

A variação no uso da estratégia apresentada para cada contexto, pode indicar que as crianças podem ter uma hierarquia de estratégias para cada exemplo. O fato que acima de $98 \%$ das respostas incluíam apenas uma estratégia não parece apoiar esta conclusão, mas isto pode ser decorrente do método. Assim, quando o experimentador iniciou a gravação logo que a criança começou a responder, esta pode ter pensado que não precisaria mencionar mais do que uma estratégia. Uma sondagem posterior relacionada à resposta poderia ter resultado em uma maior freqüência de estratégias.

A variação do contexto para respostas de emoção pode ser relacionada à hipótese de Lazarus (1966) sobre estudos de estresse. Para o autor, lidar com situações não-controláveis pode levar o sujeito a enfocar a emoção em si e, em situações-controláveis, a lidar com a situação. Esta hipótese tem sido apoiada em estudos com adultos (e.g., Blanchard-Fields \& Trion, 1988; Folkman \& Lazarus, 1980; Krantz, 1983) e em estudos com crianças (Altshuler \& Ruble, 1989; Band \& Weisz, 1988). Assim, o contexto mais incontrolável no presente estudo (morte do avô) evocou uma percentagem mais alta de respostas que implicava que alguma coisa deveria ser feita com a emoção, do que nas situações controláveis. Perder uma bola e ter um livro rasgado, resultaram em percentagens mais altas de respostas que focalizavam mudanças da situação (e.g., ganhando uma nova bola ou um novo livro). 
Um aumento de variação de respostas heterogêneas em outros contextos pode sugerir que o uso de estratégia é influenciado por outras dimensões do que a de controle. Entretanto, deveria ser notado que as crenças das crianças sobre o controle podem se desenvolver em diversos estágios. Crianças pré-escolares podem, por exemplo, acreditar que eventos podem ser controlados por pensamento ou comportamento mágico (Vikan \& Clausen, 1993). Um esclarecimento adicional sobre este ponto é portanto, dependente de uma medida de controle.

Como foi ressaltado anteriormente, o estudo de Sissel et al. (A. Vikan, comunicação pessoal, novembro de 1999) mostrou que crianças de três anos de idade podem formular algumas estratégias RE para tristeza e raiva e que essas estratégias variam em função do contexto de reações emocionais.

Os resultados do presente estudo denotam, além disso, uma expectativa do uso mais freqüente de estratégias cognitivas com a idade. A principal mudança parece ser entre cinco e sete anos. Quanto às diferenças culturais, os resultados sugeriram que as crianças norueguesas, quando comparadas às crianças brasileiras, apresentaram uma maior freqüência de respostas cognitivas e uma freqüência mais baixa de respostas sociais para raiva, mas não para tristeza. Também foi observado que as crianças brasileiras quando comparadas às norueguesas, referiam-se mais freqüentemente aos pais e menos freqüentemente aos amigos para apoio na RE.

Uma expectativa geral de que a variação nas estratégias RE deveria estar relacionada ao tipo de emoção não foi encontrada. Ambas as emoções foram classificadas como continuando apenas por um curto período de tempo, porém tristeza evocou mais respostas de apoio social dos pais do que a raiva.

O aumento em respostas cognitivas com a idade é bem conhecido em estudos sobre regulação da emoção (Harris et al 1981; McCoy \& Masters, 1985; Terwogt et al., 1986), lidando com estresse (Altshuler \& Ruble, 1989; Band \& Weisz, 1988) e lidando com a dor (e.g., Branson $\&$ Craig, 1988). Uma vez que este fato também mostra um paralelo com o desenvolvimento cognitivo em geral, seria mais surpreendente se esta mudança de idade não fosse observada. 
$\mathrm{O}$ fato de não ter havido mudanças de idade nas frequiências de outras categorias funcionais, o aumento de respostas cognitivas pode também significar que há um aumento no número de estratégias avaliáveis com a idade. Quando isto é relacionado à observação de que o contexto foi uma fonte um tanto mais saliente de variação do que a idade, a indicação de desenvolvimento foi um aumento no número de estratégias e a habilidade para variar e selecionar as mesmas de acordo com a demanda da tarefa.

Outro aspecto do desenvolvimento pode ter sido mascarado pela abrangência da interação social, que foi a categoria de escore usada com mais freqüência. Essa abrangência é indicada pelas respostas originalmente registradas como "altruísmo", que foram muito mais freqüentes nos dois grupos mais velhos do que no grupo mais novo. O procedimento também contribuiu para a abrangência, por não ter sido pedido às crianças para elaborar sobre suas respostas. Não se poderia, conseqüentemente, julgar se respostas tais como "conversar com alguém", "estar com amigos", "pedir ajuda à mãe", referiam-se a solicitações instrumentais simples ou a insights sofisticados no efeito de regulação da emoção de participação social (Rime, 1995).

Isto pode significar que o procedimento e o registro serviram para mudar o foco cognitivo relacionadas à auto-regulação e ocultar a capacidade de se relacionar e compreender os outros. Estes aspectos de desenvolvimento correspondem, respectivamente, a um objetivo individualista com característica de auto-suficiência do tipo da cultura norueguesa, e a um objetivo coletivista de solidariedade e relacionamento (Triandis, 1989), característicos de um tipo de cultura como a brasileira. A mais freqüente referência de apoio dos pais pela amostra brasileira e dos amigos pela amostra norueguesa, pode refletir, respectivamente, tendências paternalista e igualitária nas duas culturas.

A falta de diferenças nítidas de estratégias $\mathrm{RE}$ para raiva e tristeza confirma um estudo anterior com crianças de oito a doze anos que mostrou que estratégias para felicidade e medo eram diferentes entre si e também diferentes de raiva e tristeza, mas as duas últimas não se diferenciavam (Vikan \& Dias, 1996). A razão pode ser a de que muitas das situações que evocam raiva e tristeza são as mesmas, tais como 
rejeições, proibições, perder uma competição e ter um objeto seu danificado. Isto pode sugerir que para seu manuseamento, as emoções deveriam ser todas alocadas numa base central e não em bases "locais" para cada emoção como pode ser hipotetizado de um ponto de vista de emoções discretas.

Uma nota final sobre o processo de desenvolvimento é a de que há um conhecimento muito primário de estratégias RE. Quando tal conhecimento é apresentado a crianças com 3 anos (Sissel et al., conforme A. Vikan, comunicação pessoal, novembro de 1999), não deve ser devido à própria experiência da criança mas sim porque a autoobservação, memória e desenvolvimento lingüístico que são solicitados neste caso, provavelmente não estão presentes nesta idade. $\mathrm{O}$ conhecimento de RE parece ser mais um resultado da socialização, e os prováveis candidatos de dois a três anos de idade estão neste processo como mostram o desenvolvimento de expressão emocional (Malatesta-Magai, 1991), conversas entre mães e crianças sobre emoções (Dunn, Bretherton \& Munn, 1987), histórias “didáticas” de mães sobre suas respostas emocionais (Miller \& Sperry, 1987), orientação de mães da regulação da criança (Sroufe, 1996), ou combinações destes fatores. Pode-se sugerir que a codificação de tais influências para regular a vida emocional é instrumental na estabilização (internalização) que é evidente no desenvolvimento emocional da criança de idade pré-escolar (Sroufe, 1996).

\section{Referências}

Altshuler, J. A., \& Ruble, D.N. (1989). Developmental changes in children's awareness of strategies for coping with uncontrollable estresse. Child Development, 60, 1337-1349.

Band, E. B., \& Weisz, J. R. (1988). How to feel better when it feels bad: children's perspectives on coping with everyday stress. Developmental Psychology, 24, 246-253.

Blanchard-Fields, F., \& Trion, J. C. (1988). Coping strategies from the perspective of two developmental markers: age and social reasoning. Journal of Genetic Psychology, 149, 141-151.

Branson, S. M., \& Craig, K. D. (1988). Children's spontaneous strategies for coping with pain: a review. Canadian Journal of Behavioral Science, 20, 402-412. 
Brown, J. M., O'Keeffe, J., Sanders, S. H., \& Baker, B. (1986). Developmental changes in children's cognition of stressful and painful situations. Journal of Pediatric Psychology, 11, 343-357.

Curry, S. L. \& Russ, S. W. (1985). Identifying coping strategies in children. Journal of Clinical Child Psychology, 14, 61-69.

Dunn, J., \& Brown, J. (1991). Relationships, talk about feelings, and the development of affect regulation in early childhood. In J. Garber and K. A. Dodge (Orgs.), The development of emotion regulation and deregulation, (pp. 102-128). Cambridge: Cambridge University Press.

Dunn, J., Bretherton, I., \& Munn, P. (1987). Conversations about feeling states between mothers and their young children. Developmental Psychology, 23, 132-139.

Eisenberg, N., \& Fabes, R. A. (1992). Emotion and its regulation in early development. New Directions in Child Development, 55, 32-45, San Francisco: Jossey- Bass.

Folkman, S., \& Lazarus, R. S. (1980). An analysis of coping in a middle-aged community sample. Journal of Health and Social Behavioral, 21, 219239.

Fox, N. A. (1994). The development of emotion regulation. Monographs of the Society for Research in Child Development, 59, 2-3.

Garber, J. \& Dodge, K. A. (1991). The development of emotion regulation and deregulation. Cambridge: Cambridge University Press.

Harris, P. L. (1989). Children and emotion. Oxford: Blackwell

Harris, P., Olthof, T., \& Terwogt, M. M. (1981). Children's knowledge of emotion. Journal of Child Psychiatry and Psychology, 22, 247-261.

Jerret, M. D. (1985). Children and their pain experience. Children's Health Care, 14, 83-89.

Kenealy, P. (1989). Children's strategies for coping with depression. Behavioral Research and Therapy, 27, 27-34.

Kopp, C. B. (1982). Antecedents of self-regulation: a developmental perspective. Developmental Psychology, 18, 199-214.

Kopp, C. B. (1989). Regulation of distress and negative emotions: a developmental view. Developmental Psychology, 25, 343-354.

Krantz, S. E. (1983). Cognitive appraisals and problem-directed coping: a prospective study of estresse. Journal of Personality and Social Psychology, 44, 638-643.

Lazarus, R. S. (1966). Psychological estresse and the coping process. New York: McGraw Hill.

Lazarus, R. S. (1991). Emotion and adaptation. New York: Oxford University Press.

Malatesta-Magai, C. (1991). Emotional socialization: its role in personality and developmental Psychopathology. In D. Cicchetti \& S. L. Toth (Orgs.), Internalizing and externalizing expressions of dysfunction. Rochester 
Symposium on Developmental Psychopathology, Vol. 2 (pp. 203-224) Hillsdale: Lawrence Erlbaum.

McCoy, C. L., \& Masters, J. C. (1985). The development of children's strategies for the social control of emotion. Child Development, 56, 1214-1222.

Miller, P., \& Sperry, L. L. (1987). The socialization of anger and aggression. Merril-Palmer Quarterly. 33, 1-13.

Mischel, H. N., \& Mischel, W. (1983). The development of children's knowledge of self-control strategies. Child Development, 54, 603-619.

Oatley, K., \& Jenkins, J. M. (1996). Understanding emotions. Cambridge: Blackwell.

Reissland, N. (1983). Cognitive maturity and the experience of fear and pain in the hospital. Social Science and Medicine, 17, 1389-1395.

Rime, R. (1995). Mental rumination, social sharing, and the recovery from emotional exposure. In W. Pennebaker (Org.), Emotion, disclosure \& health, (pp. 77-91). Washington: American Psychological Association.

Ross, D. M., \& Ross, S. A. (1984). Childhood pain: the school-aged child's viewpoint. Pain, 20, 179-191.

Saarni, C., \& Crowley, M. (1990). The development of emotion regulation: effects on emotional state and expression. In E. A. Blechman (Org.), Emotions and the family, (pp. 23-56). Hillsdale: Lawrence Erlbaum.

Schore, A. N. (1994). Affect regulation and the origin of the self. Hillsdale: Lawrence Erlbaum

Sroufe, L. .A. (1996). Emotional development. Cambridge: Cambridge University Press

Terwogt, M. M., \& Olthof, T. (1989). Awareness and self-regulation of emotion in young children. In C. Saarni and P. L. Harris (Orgs.), Children's understanding of emotion. Cambridge: Cambridge University Press.

Terwogt, M. M., Schene, J., \& Harris, P. L. (1986). Self-control of emotional reaction by young children. Journal of Child Psychiatry and Psychology, 27, 357-366.

Thompson, R. A. (1990). Emotion and self-regulation. In R. A. Thompson (Org.), Socioemotional development, Vol. 36, (pp. 367-367). Nebraska Symposium on motivation. Lincoln: University of Nebraska Press.

Thompson, R. A. (1994). Emotion regulation: a theme in search of a definition. In N. A. Fox (Org.), The development of emotion regulation. Monographs of the Society for Research in Child Development. Vol. 59, 25-52.

Tice, D. M., \& Baumeister, R. F. (1993). Controlling anger: self-induced emotion change. In Wegner D. and J. W. Pennebaker (Orgs.), Handbook of mental control (pp. 393-409).

Englewood Cliffs: Prentice Hall.

Triandis, H. C. (1989). The self and social behavior in differing cultural contexts. Psychological Review, 96, 506-520. 
Vikan, A., \& Clausen, S. E. (1993). Freud, Piaget, or neither? Beliefs in controlling others by wishful thinking and magical behavior in young children. The Journal of Genetic Psychology, 154, 297-314.

Vikan, A., \& Clausen, C. J. (1994). Barns forstaelse. Oslo: Universitetsforlaget.

Vikan, A., \& Dias, M. G. (1996). Estratégias para o controle das emoções: um estudo transcultural entre crianças. Arquivos Brasileiros de Psicologia, $48,80-95$.

Yates, G. C. R., Yates, S. M., \& Beasley, C. J. (1987). Young children's knowledge of strategies in delay of gratification. Merril-Palmer Quarterly, $33,159-169$.

Maria da Graça Bompastor Borges Sobre os autores Dias, doutora em Psicologia do Desenvolvimento pela Universidade de Oxford (Reino Unido), é professora do Departamento de Psicologia da Universidade Federal de Pernambuco. Endereço para correspondência: Av. Beira Mar, 520 ap.81, Piedade, 54310-064, Jaboatão, PE; Fones ( $0 * * 81$ ) 3612319, 271-8272; Fax (0**81) 271-1843; email:mdias@npd.ufpe.br.

Arne Vikan, $\mathrm{PhD}$, psicólogo, é pesquisador associado (affiliated researcher) e professor de Psicologia do Centro Regional de Psiquiatria da Criança e do Jovem da Norges teknisk-naturvitenskapelige universitet NTNU (Universidade Norueguesa de Ciên- 
70 M.G.B.B. Dias et al.

cia e Tecnologia), Trondheim, Noruega. Endereço para correspondência: E-mail: arne.vikan@medisin.ntnu.no.

Sissel Elisabeth Gravås é estudante de pós-graduação do Instituto de Psicologia da Norges teknisk-naturvitenskapelige universitet - NTNU (Universidade Norueguesa de Ciência e Tecnologia), Trondheim, Noruega. Endereço para correspondência: E-mail: sisselel@stud.ntnu.no. 